\title{
Application of the Integrated Voigt Function in Analysis of Experimental Line Spectra
}

\author{
K. ILAKOVAC ${ }^{*}$ \\ Department of Physics, Faculty of Science and Mathematics, University of Zagreb, \\ Bijenička cesta 32, HR-10001 Zagreb, Croatia
}

(Received June 20, 2018; revised version April 11, 2019; in final form April 23, 2019)

\begin{abstract}
In many branches of spectroscopy, the Lorentzian and Gaussian functions are assumed to describe the intrinsic and statistical broadening effects of emission lines and of resonances. When both these broadening effects are seen, their convolutions, the Voigt functions, are used to describe shapes of the peaks. In this work, the integrated Voigt function, $V_{\mathrm{int}}$, is introduced for the calculation of the peak shapes. Also, a new model of the step function, based on physical considerations, is introduced. The model applies the same $V_{\text {int }}$ that is used to calculate the peak function. The program has been developed to analyse $K_{\alpha}$ X-ray spectra of heavy elements, measured with high-purity germanium detectors. To illustrate the method, results of analysis of two spectra are presented.
\end{abstract}

DOI: 10.12693/APhysPolA.136.107

PACS/topics: analysis of line spectra, integrated Voigt function, peak and step functions, fitting function

\section{Introduction}

This work introduces a new, accurate, simple, and low computation time method of analysis of line spectra that show intrinsic broadening according to the Lorentz distribution function and statistical broadening following the Gaussian distribution function. It has arisen in various trials to improve analysis of $K_{\alpha}$ X-ray spectra measured with high-purity germanium (HPGe) detectors. The method uses the integrated Voigt function calculated for the full width of the analysed section of spectrum and differences of its values at channel boundaries are used to calculate channel contents of the fitting function for all peaks and their step functions in the section. The model of the step function is based on physical considerations. The use of the same integrated Voigt function for the peak and for its step function reduces considerably the computer time. The method can be applied in analyses of all spectra of similar forms.

Three names, the Cauchy, the Lorentzian, and the Breit-Wigner distribution function, are used for the same function in mathematics, classical physics, and nuclear physics, respectively. In physics, this function is used to describe the natural shape of emission and resonance lines in all branches of spectroscopy. It is expressed as a function of various variables, like frequency, wave number, wavelength or energy. This work evolved in analyses of energy-dispersive spectra of $K_{\alpha}$ X-rays of high- $Z$ elements. For that reason all considerations are made in terms of energy. However, all presented relations also apply to frequency-dispersive and wavelength-dispersive spectra.

*e-mail: ilakovac@phy.hr
The Lorentzian distribution as a function of energy is given by

$$
L\left(E ; E_{0}, \Gamma\right)=\frac{1}{\pi} \frac{\Gamma / 2}{\left(E-E_{0}\right)^{2}+\Gamma^{2} / 4},
$$

where $E_{0}$ is the energy at the peak maximum and $\Gamma$ is the full-width at half-maximum (FWHM) of the peak (the intrinsic or natural width of the peak).

Results of all measurements in all branches of science are subject to statistical uncertainties and limitations of accuracy of results. They are caused by many factors, like the nature and properties of the studied object, limitations of accuracy and instability of the experimental equipment, variations of the mains voltage, noise etc. Most often many effects statistically add up and cause a distribution of results that is well described by the Gaussian distribution function,

$$
G\left(E ; E_{0}, \sigma_{0}\right)=\frac{\sigma_{0}}{\sqrt{2 \pi}} \exp \left(-\left(E-E_{0}\right)^{2} / 2 \pi \sigma_{0}^{2}\right),
$$

where $E_{0}$ is the position of the Gaussian peak and $\sigma_{0}$ - the width parameter of the Gaussian peak related to the FWHM of the Gaussian distribution by the relation $\mathrm{FWHM}=\sqrt{8 \ln 2} \sigma \approx 2.355 \sigma$.

A characteristic of the Gaussian distribution function is that it decreases very fast as $\left|E-E_{0}\right|$ increases, while the decrease of the Lorentzian is much slower. For this reason it is possible to determine natural width of a peak even when the Gaussian width is considerably larger.

In many spectra both the Lorentzian and Gaussian distributions are effective. Since they are independent, their convolution, the Voigt function, gives the joint distribution of a peak,

$$
\begin{aligned}
& V\left(E ; E_{0}, \Gamma_{0}, \sigma_{0}\right)= \\
& \quad \int_{-\infty}^{\infty} L\left(E-E^{\prime} ; E_{0}, \Gamma_{0}\right) G\left(E^{\prime} ; 0, \sigma_{0}\right) \mathrm{d} E^{\prime} .
\end{aligned}
$$


In this equation, the Gaussian function was shifted to the origin, but the value of the width $\left(\sigma_{0}\right)$ has been retained. Generally, $\sigma_{0}$ may vary as the energy changes over the natural width of a peak. But most often the peak width is much narrower than the energy of the line $\left(E_{0}\right)$ and a very good approximation is a constant value, $\sigma_{0}=\sigma\left(E_{0}\right)$, for each line in the spectrum.

Since one cannot express the integral in Eq. (3) in an algebraic form, many approximate expressions, mostly in the form of series expansions, have been invented (see e.g. Refs. [1-4]). Numerical integration is also used in analyses of simple spectra. As the spectroscopic techniques improve, the statistical effects are reduced, but they will remain to be the problem in analyses of spectra. So one can expect further work on convolution and deconvolution methods in the future.

Investigations of X-ray spectra originated with the measurements of characteristic X-ray wavelengths of elements about a century ago. Since then new methods of measurements have been introduced, techniques and precision have been greatly improved, new transitions were discovered and studied, and a vast amount of data has been accumulated.

Most data on wavelengths, line-widths, relative intensities, and other properties of transitions from highlyexcited atomic states are results of studies with the application of crystal-diffraction technique. In early times, the choice of crystals and of X-ray standards was not unique. Bearden [5] made a careful study of the different X-ray standards and units in use at the time, made measurements to determine relations among them, and proposed a general use of ångström $(\AA)$ as the unit for expessing the wavelengths and the $\mathrm{W} K_{\alpha_{1}}$ as the standard X-ray line. He presented the recalculated results for $\mathrm{X}$-ray wavelengths in $\AA$ and energies in $\mathrm{eV}$ of all elements from $\operatorname{Li}(Z=3)$ to $\operatorname{Am}(Z=95)$.

Systematic measurements of natural widths of characteristic $K_{\alpha_{1}}$ and $K_{\alpha_{2}}$ X-ray lines of 35 elements from Sb $(Z=51)$ to $\operatorname{Am}(Z=95)$ and of $K_{\beta_{1}}$ and $K_{\beta_{3}}$ $\mathrm{X}$-ray lines of 8 elements from $\mathrm{Sn}(Z=50)$ to Ta $(Z=73)$ were made by Nelson and Saunders [6] using a Cauchois-type transmission bent-crystal spectrometer. They used numerically-calculated convolutions of the Lorentzian and Gaussian distribution functions to fit the measured spectra. The results show some scatter, particularly for the $K_{\alpha_{2}}$ X-ray lines.

Kessler et al. [7] made accurate measurements of $K \mathrm{X}$ ray energies and line-widths of ten elements from silver to uranium using a newly designed double-crystal spectrometer. They introduced several innovations in the experimental arrangement, method of measurement, calibration, control of conditions, and recording of data. Their results show considerable improvements of accuracy and a better agreement with the results of theory.

Wilkinson [8] published an early study of the inversion problem, i.e. how to determine parameters of the BreitWigner (Lorentzian) distribution when its convolution with the Gaussian distribution (such as an experimental line spectrum) is known. He derived approximate expressions for the convolution function and presented in tables and diagrams the results of calculations for the natural width of the line, the total number of counts in the peak and the ratio of peak heights. He did not consider effects of distortions of the detector response function later seen as deviations from the Gaussian distribution.

Gunnink [9] developed algorithms for fitting experimental multiline spectra from samples of plutoniumbearing materials emitting $\gamma$-rays and X-rays, with the aim to determine line intensities and isotopic composition of the samples. Since natural widths of $\gamma$-ray lines are very narrow, they were represented by Gaussians, although natural widths of X-ray lines (of about $100 \mathrm{eV}$ ) were comparable to the resolution of the used $\mathrm{Ge}(\mathrm{Li})$ detectors. Therefore, the Voigt functions were used to represent the X-ray peaks. His considerations were based on the results of Wilkinson [8]. To test the algorithms, he made the measurement of the spectrum of $K_{\alpha} \mathrm{X}$-ray lines of uranium emitted from a ${ }^{235} \mathrm{~Np}$ radioactive source. In the spectrum, asymmetries were observed. They were previously seen and analysed in measurements of $\gamma$-ray spectra. The cause of the distortions of the detector response function, the long-term and the short-term tailing, were caused by imperfect collection of charge in $\mathrm{Ge}(\mathrm{Li})$ detectors. When the two tailing terms were included in the fitting function, a very good fit to the uranium $K_{\alpha}$ $\mathrm{X}$-ray spectrum was achieved.

Schulte et al. [10] made further improvements of the analysis of $K_{\alpha}$ X-ray spectra emitted by high- $Z$ atoms. They measured $K_{\alpha_{1,2}}$ spectrum of platinum also using $\mathrm{Ge}(\mathrm{Li})$ detectors. They noted that non-Gaussian nature of the two peaks is clearly seen in the Lorentzian flaring at right and left sides of the peaks. In addition to the Voigt functions representing the peaks and the previously introduced short-term and long-term exponential tails, they included the step function in the fitting function. They made the analysis of the $K_{\alpha_{1,2}}$ X-ray spectrum by two methods. First, they applied the analytic representation of the peaks by the Voigt convolutions in the approximation of Armstrong [1], adding the above quoted terms describing instrumental distortions. Second, they calculated numerical convolution of the Lorentz functions representing the two $K_{\alpha}$ peaks of platinum with experimentally determined detection response functions which were measured using $\gamma$-rays. Both methods resulted in very good fits.

The work of Schulte et al. [10] was extended by Campbell and Schulte [11]. They made careful measurements of $K_{\alpha}$ X-ray spectra of four high- $Z$ elements, ytterbium, tantalum, platinum, and lead, and analysed them by the two methods of Ref. [10]. They achieved very good results for the average widths of the two lines and for the ratio of their intensities. They also made the first determination of the intensity ratios of forbidden $K_{\alpha_{3}}$ and of $K_{\alpha_{1}}$ X-ray lines of platinum and lead.

Line spectra are regularly analysed using the Voigt functions when Lorentzian and Gaussian widths are 
comparable. For example, using a high-resolution crystal diffractometer, accurate measurements were made of diagram lines and of multiple-satellite, hypersatellite and hypersatellite-satellite $L_{\alpha_{1}}$ and $L_{\alpha_{2}}$ X-ray lines of palladium [12], of $L_{\alpha_{1,2}}$ and $L_{\beta_{1}}$ X-ray lines of zirconium, molibdenum and palladium [13], and of thorium $L_{\gamma} \mathrm{X}$ ray lines [14], produced by heavy-ion $\left(\mathrm{O}^{6+}\right)$ beams of 278.6 MeV. To interpret the observed spectra, relativistic multiconfigurational Dirac-Fock calculations were made for the lines with variable numbers of $L$ - and $M$-shell vacancies. The calculated spectra were convoluted with Gaussian distribution functions and these Voigt profiles were fitted to the experimental spectra. The parameters of the fits were the relative values of the $L$-, $M$ - and $N$ shell multiple-ionization probabilities at the moments of X-ray emissions.

\section{The apparatus and measurements}

The measurements were made using a fast-slow coincidence setup. A pair of planar HPGe detectors (supplied by ORTEC, Oak Ridge, TN, USA) was used in a close head-on geometry for the detection of X-rays (Fig. 1). Nominal size of their sensitive volumes is $200 \mathrm{~mm}^{2} \times 13 \mathrm{~mm}$ thick. The central shield was a pair of aluminium discs with copper and gold inserts and a double-taper opening. Small specs of radioactive sources were put between two thin foils of polyethylene foil, placed between the two aluminium discs, and positioned at the centre of the double-taper opening. The diameters of the double-taper opening and holes in the copper and gold inserts are 1.23, 1.75 , and $2.07 \mathrm{~mm}$, respectively.

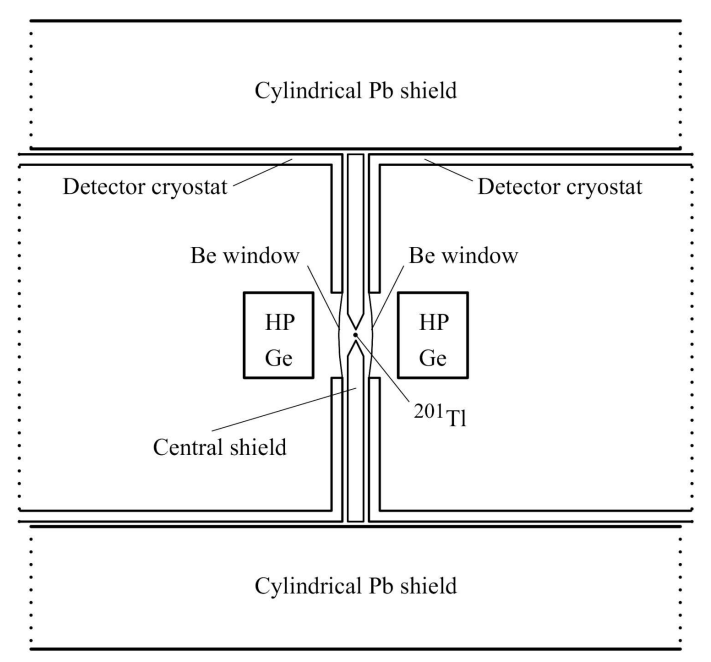

Fig. 1. Sectional view of the central part of the experimental setup.

Pulses from the detectors were fed into a fast-slow coincidence system with a three-parameter $128 \times 512 \times 512$ channel pulse-height analyzer. If a coincidental event occurred in the range of $\pm 100 \mathrm{~ns}$, the time difference was recorded in the time channel $\left(k_{0}\right)$, and amplitudes of the pulses from the detectors were recorded in the energy $\left(k_{1}\right.$ and $k_{2}$ ) channels. The data were recorded sequentially in a $\mathrm{PC}$.

Nuclear $K$-electron capture decay created $K$-shell vacancies in the tiny radioactive sources of ${ }^{179} \mathrm{Ta}$ or ${ }^{201} \mathrm{Tl}$ used in the measurements. The vacancies were followed by cascade emissions of $K_{\alpha}-L$ X-rays of hafnium and of cascades of $K_{\alpha}-L$ X-rays of mercury, respectively.

Two-dimensional $k_{1}-k_{2}$ spectra covering the region of $K_{\alpha}-L \mathrm{X}$-ray cascades were formed for in-coincidence events. Their projection onto the $k_{1}$ axis for the $L_{\gamma_{1}}$ band of channels in the case of hafnium resulted in the spectrum shown in Fig. 2, and in the case of mercury for the $L_{\alpha_{1,2}}$ band of channels in the spectrum shown in Fig. 3.

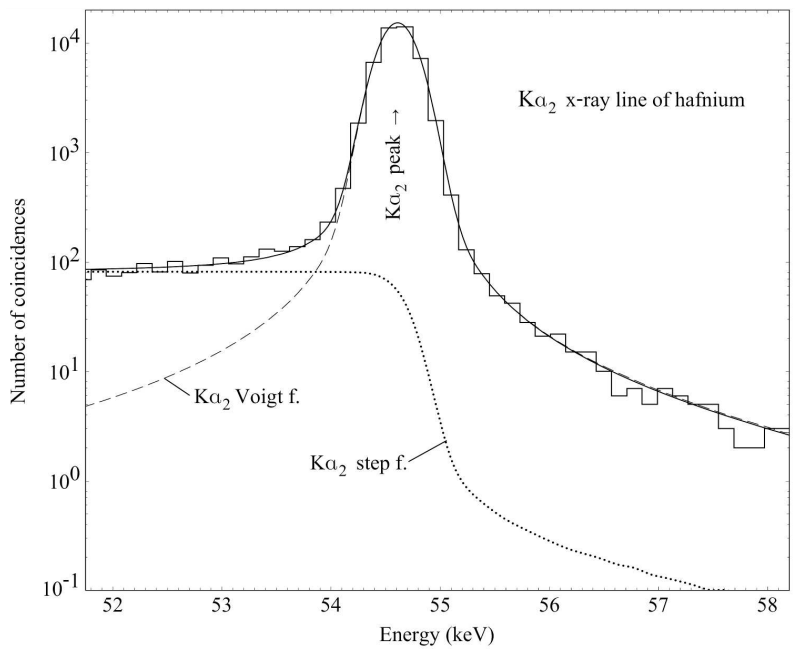

Fig. 2. $K_{\alpha} \mathrm{X}$-ray spectrum of hafnium. The histogram shows the experimental spectrum of $K_{\alpha}$ X-rays in coincidence with $L_{\gamma_{1}}$ X-rays, emitted in the decay of $K$ shell vacancy states of hafnium. The measurements were made using a pair of HPGe detectors and a pulseheight analysing system. The curves show the results of curve fitting using the integrated Voigt function for the peak and the step functions. The main values of the variable parameters are presented in the lower row in Table I.

TABLE I

Values of the main variable parameters obtained in curvefitting of the experimental spectrum of $K_{\alpha_{2}}$ X-rays of hafnium measured in coincidence with $L_{\gamma_{1}}$ X-rays. Two analyses of the spectrum were made assuming: (A) the integrated Lorentzian and (B) the integrated Voigt functions for the step function. $\Gamma_{2}$ is the natural width of the $K_{\alpha_{2}}$ X-ray line, $N_{\text {peak }}$ - the total number of pulses in the peak, $N_{\text {step }}$ - the maximal number of counts per channel of the step function, $f \times \sigma-$ the FWHM of the Gaussian function of the peak, and $\chi^{2} /$ d.f. - the reduced value of $\chi^{2}$.

\begin{tabular}{c|c|c|c|c|c}
\hline \hline & $\Gamma_{2}[\mathrm{eV}]$ & $N_{\text {peak }}$ & $N_{\text {step }}$ & $f \times \sigma[\mathrm{eV}]$ & $\chi^{2} /$ d.f. \\
\hline $\mathrm{A}$ & $35.86 \pm 1.61$ & $46989 \pm 228$ & $78.7 \pm 2.3$ & $365.5 \pm 2.3$ & $58.5 / 60$ \\
$\mathrm{~B}$ & $36.96 \pm 1.72$ & $46990 \pm 222$ & $81.6 \pm 2.6$ & $360.0 \pm 2.1$ & $52.5 / 60$
\end{tabular}


Values of the main variable parameters obtained in curve-fitting of the experimental spectrum of $K_{\alpha_{1,2}} \mathrm{X}$-rays of mercury measured in coincidence with $L_{\alpha_{1,2}}$ X-rays. Two analyses of the spectrum were made assuming: (A) the integrated Lorentzian and (B) the integrated Voigt functions for the step function. $\Gamma_{1,2}$ are the natural widths of the $K_{\alpha_{1,2}} \mathrm{X}$-ray lines, $N_{1,2}-$ the total numbers of pulses in the peaks, $N_{\text {step }, 1,2}$ - the maximal numbers of counts per channel of the step functions, $f \times \sigma_{1,2}-$ the FWHM of the Gaussian functions of the peaks, and $\chi^{2} /$ d.f. is the reduced value of $\chi^{2}$.

\begin{tabular}{c|c|c|c|c|c|c|c|c|c}
\hline \hline & $\Gamma_{1}[\mathrm{eV}]$ & $\Gamma_{2}[\mathrm{eV}]$ & $N_{1}+N_{2}$ & $N_{2} / N_{1}$ & $N_{\text {step } 1}$ & $N_{\text {step } 2}$ & $f \times \sigma_{1}[\mathrm{eV}]$ & $f \times \sigma_{2}[\mathrm{eV}]$ & $\chi^{2} / \mathrm{d} . f$. \\
\hline $\mathrm{A}$ & $54.73 \pm 2.01$ & $55.36 \pm 2.11$ & $88293 \pm 302$ & $1.020 \pm 0.045$ & $40.2 \pm 1.6$ & $40.7 \pm 1.7$ & $390.8 \pm 0.97$ & $394.2 \pm 0.98$ & $77.63 / 67$ \\
$\mathrm{~B}$ & $54.84 \pm 2.01$ & $55.43 \pm 2.23$ & $85761 \pm 307$ & $1.0312 \pm 0.042$ & $38.3 \pm 1.5$ & $39.0 \pm 1.7$ & $390.7 \pm 0.97$ & $394.0 \pm 0.98$ & $76.49 / 67$
\end{tabular}

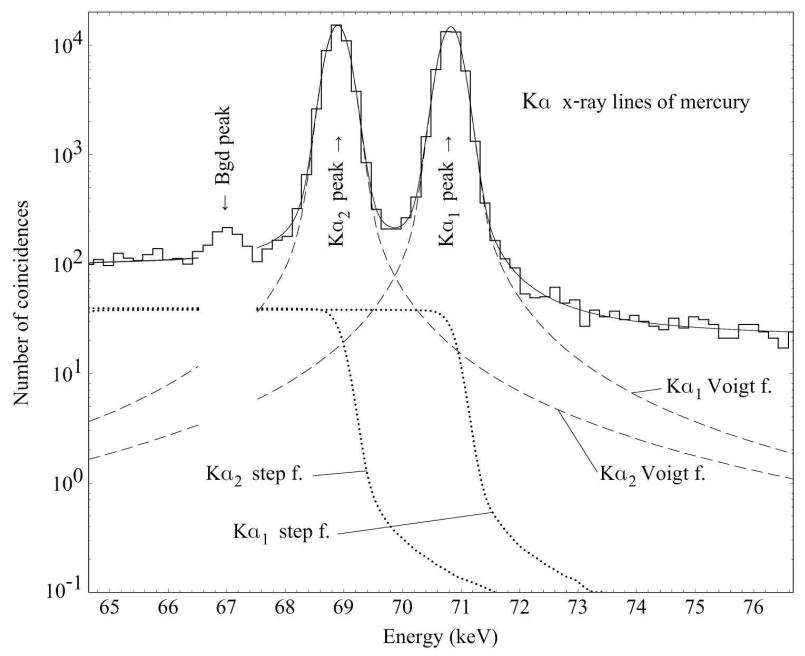

Fig. 3. $K_{\alpha} \mathrm{X}$-ray spectrum of mercury. The histogram shows the experimental spectrum of $K_{\alpha}$ X-rays in coincidence with $L_{\alpha} \mathrm{X}$-rays, emitted in the decay of the $K$-shell vacancy states of mercury. As in the case of Hf, the measurements were made using a pair of HPGe detectors and a pulse-height analysing system. The curves show the results of curve fitting using the integrated Voigt functions for two peaks and their step functions. The main values of the variable parameters of the fit are presented in the lower row in Table II. Note: Five channels around the little "Background peak" have been omitted in the curve-fitting procedure to avoid its effects on the results. A constant background of $21 \pm 1$ pulses was included in the fitting function (full line).

\section{The integrated Voigt function}

The Voigt distribution is most often applied in analyses of experimental line spectra with the aim to determine the values of characteristic parameters (peak positions, their intensities and/or widths) of spectral lines which are broadened by imperfect operation of experimental equipment, such as the Doppler effect, temperature variations, instabilities of energy supply, and other effects. Generally, the curve-fitting procedures are applied to solve the problem, i.e. the sought after spectral parameters and other parameters that influence the spectrum are assumed, the fitting curve is calculated, and a comparison is made to the experimental spectrum. The standard criterion of quality of the fit is the value of $\chi^{2}$. Various computer routines are used to find the best fit (minimum $\chi^{2}$ ) and to determine the spectral parameters based on the model of the fitting function. Since this procedure requires a large number computations of the Voigt function and much computation time, many approximations have been invented to reduce computation time and to achieve required accuracy of results.

One way to speed up the computations is the use of integrated Voigt function. Brüggemann and Bollig [15] introduced the integrated Voigt function for spectral synthesis and band integration of multiline spectra. For a given set of parameters, one computes the Voigt functions of the peaks and forms the integrated spectrum. Simple differences between pairs of cuts in the integrated spectrum give the intensity of the corresponding band of lines. To calculate the Voigt functions, they used a series expansion of the complex Voigt function.

Quine and Abrarov [16] introduced the spectrally integrated Voigt function for modelling radiation transfer by absorption and emission in gas mixtures (e.g. in lower layers of the Earth stratosphere). Since large numbers of lines are present in the spectra, the standard line-byline method requires much computer time if high resolution is required. They calculate the spectrally integrated Voigt function for its sections around the peaks (not for the entire analysed spectrum). They show that by using the spectrally integrated Voigt function with low resolution, results of high-resolution line-by-line method are achieved with substantially reduced computer time. They also analysed the Brüggemann and Bollig approximation of the Voigt function and found that its values are erroneous for small absolute values of the complex variable $z$.

Both these works treat the problems of intensities of lines or bands of lines and do not consider analysis of shapes of lines.

In this work, the integrated Voigt function is introduced with the purpose to analyse the peak shapes, in particular to determine the line-widths of high- $Z K_{\alpha} \mathrm{X}$ ray lines.

The integrated and normalized Voigt function of a peak is defined by:

$$
\begin{aligned}
& V_{\text {int }}\left(E ; E_{0}, \Gamma_{0}, \sigma_{0}\right)= \\
& \quad \int_{-\infty}^{E} V\left(E^{\prime} ; E_{0}, \Gamma_{0}, \sigma_{0}\right) \mathrm{d} E^{\prime} / \int_{-\infty}^{\infty} V\left(E^{\prime} ; E_{0}, \Gamma_{0}, \sigma_{0}\right) \mathrm{d} E^{\prime} .
\end{aligned}
$$


At $E=-\infty, V_{\text {int }}=0$. As $E$ increases, it rises very slowly up to several FWHM below $E_{0}$. At $E=E_{0}, V_{\text {int }}=0.5$ and the increase of $V_{\text {int }}$ is fastest. For higher values of $E$, the rate of increase slows down. Finally, for $E=\infty, V_{\text {int }}$ reaches its limiting value 1 .

In numerical calculations, the function $V_{\text {int }}$ is used in a limited interval of the variable $E$ in the range of the peak.

\section{The step function}

A distortion due to the non-perfect operation of experimental systems are the so-called steps. They are seen in experimental spectra as different numbers of counts per channel at left and right sides of peaks. Their heights and shapes depend on the experimental arrangement used in the measurement. In the sample spectra shown in Figs. 2 and 3 , the steps are about a hundred times lower than the peak heights. In the following considerations, a simple interpretation of their appearance is presented. Figure 4 illustrates main points of the approach. A certain process or series of processes (the Compton scattering, escape of electron ejected by the incoming radiation from the sensitive volume of the detector, incomplete collection of charges, neutralization of electron-hole pairs during the charge collection in the detector etc.) reduce pulse heights by a certain amount. In that way a small Voigt peak of the same Voigt shape and width as the main peak appears below the main peak at a position corresponding to an energy $E_{c}$. Other processes give rise to such peaks at different positions. As a result, a continuum of small peaks forms below and adjacent to the main peak. The assumption that the small peaks are of the same Voigt shape and width as the main peak seems to be reasonable because energy changes over the width of the continuum are small in comparison to the energy $E_{0}$. To simplify the calculations, it is also assumed that the continuum of small peaks is of a certain width, $E_{w}$, and of a constant height. These approximations are nearly correct and seem to be good in the spectra shown in Figs. 2 and 3 and in other analysed spectra of $K_{\alpha} \mathrm{X}$-ray emissions from heavy elements. A modification of the program could be introduced to define the profile of the continuum.

Let us suppose that peaks in a spectrum with several lines at peak positions $\left(E_{0}\right)_{j}=E_{j}$ are represented by the Voigt functions $V\left(E ; E_{j}, \Gamma_{j}, \sigma_{j}\right)$ and their integrated forms $V_{\text {int }}\left(E ; E_{j}, \Gamma_{j}, \sigma_{j}\right)$. Consider a spectrum with several lines at positions $\left(E_{0}\right)_{j}=E_{j}$. For each peak, the above discussed model of the appearance of the step functions allows their simple calculation using the $V_{\text {int }}\left(E ; E_{j}, \Gamma_{j}, \sigma_{j}\right)$ functions. Consider the Voigt function of a peak at the energy $E_{j}$ and the continuum of small peaks below the peak in the energy range $E_{j}-E_{w}$ to $E_{j}$, where $E_{w}$ is the width of the continuum of small peaks (see Fig. 4). An energy interval $\Delta E_{c}$ at an energy $E_{c}$ in that range gives a contribution $V\left(E ; E_{c}, \Gamma_{j}, \sigma_{j}\right) \Delta E_{c}$ at the position $E$, where $\Gamma_{j}$ and $\sigma_{j}$ are the widths of the main peak (in the approximation explained above).

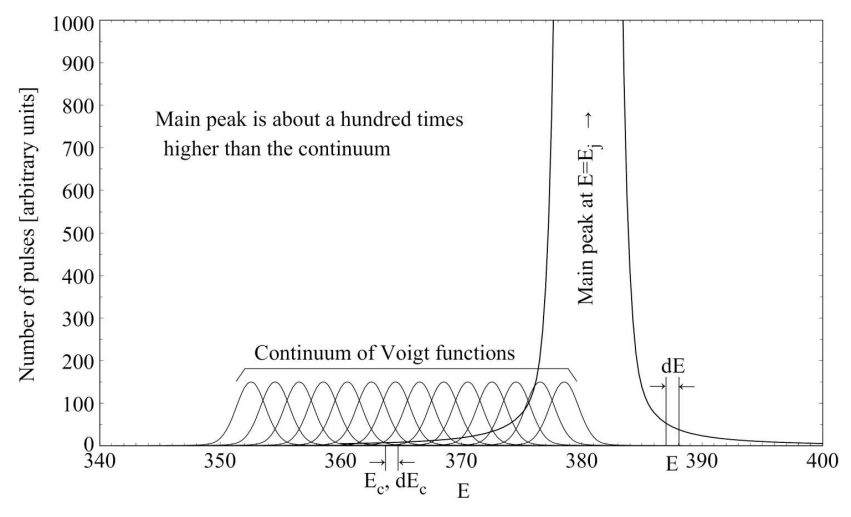

Fig. 4. Model of the step function. Complete absorption of energy of incident photons in the sensitive volume of the HPGe detector gives rise to the main peak of the Voigt profile at the position $E_{j}$. Due to the incomplete absorption, pulses of reduced amplitude appear below the main peak. In the new model, it is assumed that a continuum of small peaks of the same form as of the main peak forms adjacent to the main peak. A small peak at the position $E_{c}$ gives rise to a small contribution at a position $E$ of the spectrum. The sum of contributions over the width of the continuum $\left(E_{w}\right)$ gives rise to the step function. A simple way to calculate the step function with the use of the integrated Voigt function is presented in the text.

Calculations of integrals over the range of the continuum can be complicated. However, application of the integral Voigt function $V_{\text {int }}\left(E ; E_{j}, \Gamma_{j}, \sigma_{j}\right)$ offers a very simple solution. Namely, since the shapes of the small Voigt peaks in the continuum are assumed to be the same as that of the main peak at the energy $E_{j}$,

$V\left(E ; E_{c}, \Gamma_{j}, \sigma_{j}\right)=V\left(E+E_{j}-E_{c} ; E_{j}, \Gamma_{j}, \sigma_{j}\right)$, since differences $E-E_{c}$ and $E+E_{j}-E_{c}-E_{j}$ are equal. Therefore,

$\int_{E_{j}-E_{w}}^{E_{j}} V\left(E ; E_{c}, \Gamma_{j}, \sigma_{j}\right) \mathrm{d} E_{c}=\int_{E}^{E+E_{w}} V\left(E^{\prime} ; E_{j}, \Gamma_{j}, \sigma_{j}\right) \mathrm{d} E^{\prime}$.

where the approximation of constant continuum of small peaks has been applied. The integral at the right side of Eq. (6) is equal to the difference $V_{\text {int }}\left(E+E_{c} ; E_{j}, \Gamma_{j}, \sigma_{j}\right)-$ $V_{\text {int }}\left(E ; E_{j}, \Gamma_{j}, \sigma_{j}\right)$.

\section{Numerical calculation of the fitting function}

In the calculation of the fitting function three linear scales are used: the channel numbers of the experimental spectrum $k$, the energy scale $E$, and the scale of the variable $z$ that is used in the subroutine for the calculation of the integrated Voigt function. The linear relations among them are defined by the positions of two peaks of the analysed spectrum. If only one peak is present (because a narrow section of the spectrum is analysed), then a peak in the lower or in the higher part of the spectrum of the same measurement is used. 
The calculations of the fitting functions should be made using at least 20 values of the variable $z$ per channel of the experimental spectrum.

The computer program is composed of two parts, the main program and the subroutine. The main program starts the calculations using input data, an initial set of variable parameters, $v_{i}$, and of values of their shifts, $\Delta v_{i}, i=1,2, \ldots, n_{p}$, where $n_{p}$ is the number of variable parameters. In the subroutine, for each variable parameter, the values of $\chi^{2}$ are calculated for $v_{i}-\Delta v_{i}, v_{i}$ and $v_{i}+\Delta v_{i}\left(3 n_{p}\right.$ values of $\left.\chi^{2}\right)$. These values are transferred to the main program where on the basis of gradients of $\chi^{2}$ a new set of $v_{i}$ and $\Delta v_{i}$ is determined that is used in the next round of calculations in the search for the best fit. The calculations proceed until the minimum of $\chi^{2}$ is reached.

The main task of the subroutine is the calculation of values of the fitting curves and of $\chi^{2}$. These calculations proceed in the following way. For a set of variable parameters transferred from the main program and for each peak in the experimental spectrum, using Eqs. (3) and (4), the values of the Lorentzian and Gaussian functions are calculated for $z_{i}=i \Delta z$, where $i=0,1,2, \ldots, N$ and $\Delta z$ is the step of the scale. The scale should cover a broader range of energy than the experimental spectrum to allow for the calculation of the relatively broad Lorentz peaks and the steps of peaks. When the functions decrease below $10^{-12}$ times their maximum values, they are set at these limiting values. The convolution of the Lorentzian and Gaussian functions, the Voigt function, is calculated using Eq. (5) and the integrated and normalized Voigt functions using Eq. (6).

The main result of the present article is the application of the integrated and normalized Voigt functions in the calculation of the channel contents of the fitting functions. Each line is represented by a Voigt function of the peak and by its step function. The sum of fitting functions of all lines in the spectrum and of a constant background is used in the calculation of $\chi^{2}$ for the given set of variable parameters.

The energy of the middlepoint of the channel $k$, $E_{k}$, and the channel width, $\Delta E$, are determined on the basis of the positions of two peaks in the spectrum. Using the relation of the energy and $z$ scales and cubic interpolation, the values of the integrated Voigt function of the peak $j, V_{\text {int }}\left(E_{k}-\right.$ $\left.\Delta E / 2 ; E_{0, j}, \Gamma_{j}, \sigma_{j}\right)$ and $V_{\text {int }}\left(E_{k}+\Delta E / 2 ; E_{0, j}, \Gamma_{j}, \sigma_{j}\right)$ are calculated. The content of channel $k$ of the peak $j$ is given by

$$
\begin{aligned}
& n_{\text {peak }}(j, k)=N(j)\left[V_{\text {int }}\left(E_{k}+\Delta E / 2 ; E_{0, j}, \Gamma_{j}, \sigma_{j}\right)\right. \\
& \left.\quad-V_{\text {int }}\left(E_{k}-\Delta E / 2 ; E_{0, j}, \Gamma_{j}, \sigma_{j}\right)\right],
\end{aligned}
$$

where $N(j)$ (a variable parameter) is the total number of counts in the peak $j$. It should be noted that the application of $V_{\text {int }}$ resolves accurately the histogram problem.
The content of the channel $k$ of the step function associated with the peak $j$ in the approximation of constant continuum of small peaks is

$$
\begin{aligned}
& n_{\text {step }}(j, k)=N_{\text {step }}(j)\left[V_{\text {int }}\left(E+E_{w} ; E_{j}, \Gamma_{j}, \sigma_{j}\right)\right. \\
& \left.\quad-V_{\text {int }}\left(E ; E_{j}, \Gamma_{j}, \sigma_{j}\right)\right],
\end{aligned}
$$

where $N_{\text {step }}(j)$ is approximately the maximal number of counts per channel of the step function of the peak $j$. If this approximation is not satisfactory and a modulation of continuum of small peaks is known, it could be simply introduced in this equation.

The calculated content for the channel $k$ is given by

$$
n_{\text {calc }}(k)=\sum_{j}\left[n_{\text {peak }}(j, k)+n_{\text {step }}(j, k)\right]+n_{\text {bgd }},
$$

where $n_{\text {bgd }}$ is the background per channel. $n_{\text {calc }}(k)$ is the fitting function which is used in the calculation of $\chi^{2}$.

Attempts to include $E_{w}$ in the set of variable parameters were not successful because the dependence of $\chi^{2}$ on $E_{w}$ does not show a well defined minimum. If the value of $E_{w}$ is too small, the minimum value of $\chi^{2}$ is large (mainly due to contributions in low channels of the spectrum). As $E_{w}$ is increased, the minimum value of $\chi^{2}$ decreases. These changes of $E_{w}$ affect negligibly the results of variable parameters at minimum $\chi^{2}$. When the decrease becomes negligible, the results of variable parameters and of $\chi^{2}$ are considered to be final.

All calculations of the fitting function and of $\chi^{2}$ are made in double precision. Since the expressions used for the calculations of $V_{\text {int }}$ bear no approximations, its accuracy is about 1 part in $10^{12}$. The values of $V_{\text {int }}$ at the channel boundaries are calculated by cubic interpolation, so their accuracy is slightly lower. The whole change of $V_{\text {int }}$ across a peak is split by the channel boundaries into about 100 steps (the channels), so the accuracy of the calculated channel contents is about 1 part in $10^{9}$. Therefore, if the assumption that the shape of the peaks is Voigtian, the new method of their calculation using $V_{\text {int }}$ and of $\chi^{2}$ is very accurate, about 1 part in $10^{9}$.

The accuracy of calculation of the step functions is considerably lower because of the approximation that the profile of the continuum of little peaks is constant and of a certain width. However, this approximation is considered to have a small effect on results for peak parameters because the step functions are about 100 times lower than the peak heights.

To check the new method, its comparison to peak fitting using Gaussian functions was considered, but the detailed study of Wegrzynek et al. [17] made it pointless. Another check was made, the calculation of the step function using the integrated Lorentzian function instead of the integrated Voigt function. The comparison of results for the spectrum shown in Fig. 2 is given in Table I and for the spectrum shown in Fig. 3 in Table II. Lower values of $\chi^{2}$ have been obtained, but the differences are small because, as mentioned above, the step functions are much smaller than the main peaks. 


\section{Conclusions}

Previous publications on the use of integrated Voigt function study band integration of multiline spectra [15] and calculation of line intensities when spectral resolution is varied [16]. This article introduces the application of the integrated Voigt function in analysis of shapes of line spectra with a particular attention to line widths. When numerical convolution is used to calculate the Voigt functions for fitting experimental line spectra, an easy further step is to calculate the integrated Voigt functions and their values at channel boundaries of the experimental spectrum. The differences of these values give the calculated channel contents of both the peak and step functions. For each line in the spectrum and a given set of variable parameters, just one calculation of the integrated Voigt function is needed. Using these functions, accurate fitting functions are formed for the calculation of values of $\chi^{2}$ in the search of the best fit to experimental spectrum and for final values of the variable parameters. The application of the integrated Voigt functions and their differences accurately solves the histogram problem. Based on physical considerations, a simple model of the step function has been introduced. For illustration, results of analyses of two experimental spectra are presented in Figs. 2 and 3. They are the spectrum $K_{\alpha}$ X-rays in coincidence with $L_{\gamma_{1}}$ X-rays of hafnium and the spectrum $K_{\alpha} \mathrm{X}$-rays in coincidence with $L_{\alpha} \mathrm{X}$-rays of mercury. The measurements were made with a pair of high-purity germanium detectors and a coincidence system. The results of the analyses indicate that application of the integrated Voigt functions in analyses of experimental line spectra is an accurate, fast, and easily usable method.

\section{Acknowledgments}

The author expresses his thanks for the hospitality of the Department of Physics, Faculty of Science and Mathematics of the University of Zagreb, where he did this work as emeritus professor.

\section{References}

[1] B.H. Armstrong, J. Quant. Spectrosc. Radiat. Transf. 7, 61 (1976).

[2] A.K. Hui, B.H. Armstrong, A.A. Wray, J. Quant. Spectrosc. Radiat. Transf. 19, 509 (1978).

[3] F. Schreier, J. Quant. Spectrosc. Radiat. Transf. 48, 743 (1992).

[4] K.L. Letchworth, D.C. Benner, J. Quant. Spectrosc. Radiat. Transf. 107, 173 (2007).

[5] A. Bearden, Rev. Mod. Phys. 39, 78 (1967).

[6] G. Nelson, B. Saunders, J. Phys. Coll. 32, C4-97 (1971).

[7] E.G. Kessler Jr., R.D. Deslattes, D. Girard, W. Schwitz, L. Jacobs, O. Renner, Phys. Rev. A 26, 2696 (1982).

[8] D.L. Wilkinson, Nucl. Instrum. Methods 95, 259 (1971).

[9] R. Gunnink, Nucl. Instrum. Methods 143, 145 (1977).

[10] C.W. Schulte, H.H. Jorch, J.L. Campbell, Nucl. Instrum. Methods 174, 549 (1980).

[11] J.L. Campbell, C.W. Schulte, Phys. Rev. A 22, 609 (1980).

[12] M. Czarnota, D. Banaš, M. Breset, D. Chmielewska, J.Cl. Dousse, J. Hoszowska, Y.P. Maillard, O. Mauron, M. Pajek, M. Polasik, P.A. Raboud, J. Rzadkiewicz, K. Słabkowska, Z. Sujkowski, Phys. Rev. A 81, 064702 (2010).

[13] M. Czarnota, D. Banaš, M. Berset, D. Chmielewska, J.Cl. Dousse, J. Hoszowska, Y.P. Maillard, O. Mauron, M. Pajek, M. Polasik, P.A. Raboud, J. Rzadkiewicz, K. Słabkowska, Z. Sujkowski, Phys. Rev. A 88, 052505 (2013).

[14] K. Słabkowska, J. Starosta, E. Wéder, Ł. Syrocki, M. Polasik, Phys. Rev. A 96, 012506 (2017).

[15] D. Brüggemann, M. Bollig, J. Quant. Spectrosc. Radiat. Transf. 48, 111 (1992).

[16] B.M. Quine, S.M. Abrarov, J. Quant. Spectrosc. Radiat. Transf. 127, 37 (2013).

[17] D. Wegrzynek, A. Markowicz, A. Mendoza Cuevas, X-Ray Spectrom. 30, 403 (2001). 